\title{
UNIFORM BLOW-UP ESTIMATES FOR NONLINEAR HEAT EQUATIONS AND APPLICATIONS
}

\author{
FRANK MERLE* AND HATEM ZAAG ${ }^{\dagger}$
}

We consider the following nonlinear heat equation

$$
\left\{\begin{aligned}
u_{t} & =\Delta u+|u|^{p-1} u \\
u(0) & =u_{0}
\end{aligned}\right.
$$

where $u:(x, t) \in \mathbb{R}^{N} \times[0, T) \rightarrow \mathbb{R}$

$$
1<p,(N-2) p<N+2 \text { and either } u_{0} \geq 0 \text { or }(3 N-4) p<3 N+8 \text {. }
$$

More general vector-valued heat equations can be considered with similar results (see [MZ99] for more details) :

$$
\left\{\begin{aligned}
u_{t} & =\Delta u+F(|u|) u \\
u(0) & =u_{0}
\end{aligned}\right.
$$

where $u: \Omega \times[0, T) \rightarrow \mathbb{R}^{M}, p$ satisfies $(0.2), \Omega=\mathbb{R}^{N}$ or $\Omega$ is a smooth bounded convex domain of $\mathbb{R}^{N}, F(|u|) \sim|u|^{p-1}$ as $|u| \rightarrow+\infty$, and $M \in \mathbb{N}$.

We are interested in the blow-up phenomenon for (0.1). Many authors have been interested in this topic. Let us mention for instance Friedman [Fri65], Fujita [Fuj66], Ball [Bal77], Bricmont and Kupiainen [BKL94], Chen and Matano [CM89], Galaktionov and Vázquez [GV95], Giga and Kohn [GK89], [GK87], [GK85], Herrero and Velázquez [HV93], [HV92].

In the following, we consider $u(t)$ a blow-up solution of $(0.1)$ and denote its blowup time by $T$. We aim at finding sharp uniform estimates at blow-up and specifying the blow-up behavior of $u(t)$. Such a study is done considering equation (0.1) in its self-similar form : for all $a \in \mathbb{R}^{N}$, we define

$$
y=\frac{x-a}{\sqrt{T-t}}, s=-\log (T-t), w_{a, T}(y, s)=(T-t)^{\frac{1}{p-1}} u(x, t)
$$

Therefore, $w_{a, T}=w$ satisfies $\forall s \geq-\log T, \forall y \in \mathbb{R}^{N}$ :

$$
\frac{\partial w}{\partial s}=\Delta w-\frac{1}{2} y \cdot \nabla w-\frac{w}{p-1}+|w|^{p-1} w .
$$

Let us introduce the following Lyapunov functional associated with (0.5) :

$$
E(w)=\int_{\mathbb{R}^{N}}\left(\frac{1}{2}|\nabla w|^{2}+\frac{1}{2(p-1)}|w|^{2}-\frac{1}{p+1}|w|^{p+1}\right) \rho(y) d y
$$

*Département de mathématiques, Université de Cergy-Pontoise, 2 avenue Adolphe Chauvin, B.P. 222, Pontoise, 95302 Cergy-Pontoise cedex, France (merle@math.pst.u-cergy.fr).

†Département de mathématiques et applications, École Normale Supérieure, 45 rue d'Ulm, 75230 Paris cedex 05, France (Hatem.Zaag@ens.fr). 
where $\rho(y)=\frac{e^{-\frac{|y|^{2}}{4}}}{(4 \pi)^{N / 2}}$.

The question is to estimate $w_{a, T}(s)$ as $s \rightarrow+\infty$, uniformly with respect to $a$, whether $a$ is a blow-up point or not ( $a$ is called a blow-up point if there exists $\left(a_{n}, t_{n}\right) \rightarrow(a, T)$ such that $\left.\left|u\left(a_{n}, t_{n}\right)\right| \rightarrow+\infty\right)$.

Giga and Kohn showed that self-similar variables are convenient for describing the blow-up rate in the following sense : there exists $\epsilon_{0}\left(u_{0}\right)>0$ such that $\forall s \geq s_{0}^{*}\left(u_{0}\right)$,

$$
\epsilon_{0} \leq|w(s)|_{L^{\infty}} \leq \frac{1}{\epsilon_{0}} .
$$

We first aim at sharpening this result in order to obtain compactness properties in our problem.

1. A Liouville Theorem for equation (0.5). We are interested in classifying all global and bounded solutions of (0.5), for all subcritical $p$ :

$$
p>1 \text { and }(N-2) p<N+2 \text {. }
$$

We claim the following :

Theorem 1. A Liouville Theorem for equation (0.5). Assume (1.1) and consider $w$ a solution of (0.5) defined for all $(y, s) \in \mathbb{R}^{N} \times \mathbb{R}$ such that $\forall(y, s) \in$ $\mathbb{R}^{N} \times \mathbb{R},|w(y, s)| \leq C$. Then, either $w \equiv 0$, or $w \equiv \kappa$ or $w(y, s)= \pm \varphi\left(s-s_{0}\right)$ where $\kappa=(p-1)^{-\frac{1}{p-1}}, s_{0} \in \mathbb{R}$ and $\varphi(s)=\kappa\left(1+e^{s}\right)^{-\frac{1}{p-1}}$.

REMARK. $\varphi$ is in fact an $L^{\infty}$ connection between two critical points of $(0.5): \kappa$ and 0 . Indeed,

$$
\dot{\varphi}=-\frac{\varphi}{p-1}+\varphi^{p}, \varphi(-\infty)=\kappa, \varphi(+\infty)=0 .
$$

REMARK. A similar classification result can be obtained with a solution $w$ defined only on $\left(-\infty, s^{*}\right)$ (see [MZ98]).

Theorem 1 has the following corollary :

Corollary 1. A Liouville Theorem for equation (0.1). Assume (1.1) and consider $u$ a solution of $(0.1)$ defined for all $(x, t) \in \mathbb{R}^{N} \times(-\infty, 0)$ such that $\forall(x, t) \in \mathbb{R}^{N} \times(-\infty, 0),|u(x, t)| \leq C(-t)^{-\frac{1}{p-1}}$. Then, either $u \equiv 0$, or $u(x, t)=$ $\pm \kappa\left(T^{*}-t\right)^{-\frac{1}{p-1}}$ for some $T^{*} \geq 0$.

The proofs can be found in [MZ99] and [MZ98]. The key tools in the proof are the following :

i) A classification of all possible linear behaviors of $w(s)$ as $s \rightarrow-\infty$ in $L_{\rho}^{2}\left(\mathbb{R}^{N}\right)$ $\left(L_{\text {loc }}^{\infty}\left(\mathbb{R}^{N}\right)\right)$,

ii) The following geometric transformations which keeps (0.5) invariant :

$$
w(y, s) \rightarrow w_{a, b}(y, s)=w\left(y+a e^{\frac{s}{2}}, s+b\right),
$$

where $a \in \mathbb{R}^{N}$ and $b \in \mathbb{R}$,

iii) A blow-up criterion for (0.5) used for solutions close to the constant point $\kappa$ (This criterion is also a blow-up criterion for (0.1) via the transformation (0.4)) : 
If for some $s_{0} \in \mathbb{R}, I\left(w\left(s_{0}\right)\right)>0$ where

$$
I(w)=-2 E(w)+\frac{p-1}{p+1}\left(\int_{\mathbb{R}^{N}}|w(y)|^{2} \rho(y) d y\right)^{\frac{p+1}{2}},
$$

then $w(s)$ blows up in finite time.

REMARK. This criterion is sharp for solutions near constants. Indeed, if $w\left(s_{0}\right) \equiv$ $C_{0}$, then

$$
w \text { blows-up in finite time } \Leftrightarrow\left|C_{0}\right|>\kappa \Leftrightarrow I\left(C_{0}\right)>0 .
$$

REMARK. The proof of the Liouville Theorem strongly relies on the existence of a Lyapunov functional for equation (0.5) and can not be extended to other systems where the nonlinearity is not a gradient. In [Zaa], we go beyond this restriction and introduce new tools to prove a Liouville Theorem of the same type for the following system

$$
u_{t}=\Delta u+v^{p}, \quad v_{t}=\Delta v+u^{q} .
$$

2. Localization at blow-up. We assume again (0.2). The estimate (0.6) of Giga and Kohn gives compactness in the problem. Using a compactness procedure in the singular zone of $\mathbb{R}^{N}$ (which is, say $\left\{y|| w(y, s) \mid \geq \frac{\kappa}{2}\right\}$ ), we find a solution satisfying the hypotheses of Theorem 1 . Therefore, $\Delta w$ is small with respect to $|w|^{p}$ in this singular zone (or equivalently, $\Delta u$ is small with respect to $|u|^{p}$ ). A subcritical localization procedure introduced by Zaag [Zaa98] (under the level of the constant $\kappa)$ allows us to propagate this estimate towards the intermediate zone between the singular and the regular one. We claim the following :

THEOREM 2. Comparison with the associated ordinary differential equation. Assume (0.2) and consider $T \leq T_{0}$ and $\left\|u_{0}\right\|_{C^{2}\left(\mathbb{R}^{N}\right)} \leq C_{0}$. Then, $\forall \epsilon>0$, there is $C\left(\epsilon, C_{0}, T_{0}\right)$ such that $\forall(x, t) \in \mathbb{R}^{N} \times[0, T)$,

$$
\left.\left.\left|u_{t}-\right| u\right|^{p-1} u|\leq \epsilon| u\right|^{p}+C .
$$

REMARK. This way, we prove that the solution of the PDE (0.1) can be uniformly and globally in space-time compared to a solution of an ODE (localized by definition). Note that the condition $u(0) \in C^{2}\left(\mathbb{R}^{N}\right)$ is not restrictive because of the regularizing effect of the Laplacian.

REMARK. Many striking corollaries can be derived from this theorem. It implies in particular that no oscillation is possible near a blow-up point $a$, and that $|u(x, t)| \rightarrow$ $+\infty$ as $(x, t) \rightarrow(a, T)$. Moreover, $\forall \epsilon_{0}>0$, there exists $t_{0}\left(\epsilon_{0}\right)<T$ such that for all $b \in \mathbb{R}^{N}$, if $|u(b, t)| \leq\left(1-\epsilon_{0}\right) \kappa(T-t)^{-\frac{1}{p-1}}$ for some $t \in\left[t_{0}, T\right)$, then $b$ is not a blow-up point (this specifies more precisely a former result by Giga and Kohn where $\left.t_{0}=t_{0}\left(\epsilon_{0}, a\right)\right)$.

3. Optimal $L^{\infty}$ estimates at blow-up. We still assume (0.2). Using estimate (0.6) and the Liouville Theorem, we make a compactness argument to get the following sharp estimates :

Theorem 3. $L^{\infty}$ refined estimates for $w(s)$. Assume that (0.2) holds. Then, there exist positive constants $C_{i}$ for $i=1,2,3$ such that if $u$ is a solution of (0.1) 
which blows-up at time $T$ and satisfies $u(0) \in C^{3}\left(\mathbb{R}^{N}\right)$, then $\forall \epsilon>0$, there exists $s_{1}(\epsilon) \geq-\log T$ such that $\forall s \geq s_{1}, \forall a \in \mathbb{R}^{N}$,

$$
\begin{array}{rlrl}
\left\|w_{a, T}(s)\right\|_{L^{\infty}} & \leq \kappa+\left(\frac{N \kappa}{2 p}+\epsilon\right) \frac{1}{s}, \quad\left\|\nabla w_{a, T}(s)\right\|_{L^{\infty}} \leq \frac{C_{1}}{\sqrt{s}} \\
\left\|\nabla^{2} w_{a, T}(s)\right\|_{L^{\infty}} & \leq \frac{C_{2}}{s}, & \left\|\nabla^{3} w_{a, T}(s)\right\|_{L^{\infty}} \leq \frac{C_{3}}{s^{3 / 2}}
\end{array}
$$

Remark. In the case $N=1$, Herrero and Velázquez (Filippas and Kohn also) proved some related estimates, using a Sturm property introduced in particular by Chen et Matano (the number of space oscillations is a decreasing function of time).

REMARK. The constant $\frac{N \kappa}{2 p}$ is optimal (see Herrero and Velázquez, Bricmont and Kupiainen, Merle and Zaag).

4. Different notions of blow-up profiles and the stability question. We assume (0.2). We consider $a \in \mathbb{R}^{N}$, a blow-up point of $u(t)$, solution of $(0.1)$. From translation invariance, we can assume $a=0$. We would like to know whether $u(t)$ (or $w_{0, T}(s)$ defined in (0.4)) has a universal behavior or not, as $t \rightarrow T$ (or $s \rightarrow+\infty$ ).

Filippas, Kohn, Liu, Herrero et Velázquez prove that $w$ behaves in two distinct ways :

- either $\forall R>0, \sup _{|y| \leq R}\left|w(Q y, s)-\left[\kappa+\frac{\kappa}{2 p s}\left(l-\frac{1}{2} \sum_{k=1}^{l} y_{k}^{2}\right)\right]\right|=O\left(\frac{1}{s^{1+\delta}}\right)$ as $s \rightarrow$ $+\infty$, for some $\delta>0$ where $l \in\{1, \ldots, N\}, Q$ is a $N \times N$ orthogonal matrix and $I_{l}$ is the $l \times l$ identity matrix.

- or $\forall R>0, \sup _{|y| \leq R}|w(y, s)-\kappa| \leq C(R) e^{-\lambda_{0} s}$ for some $\lambda_{0}>0$.

From a physical point of view, these results do not tell us much about the transition between the singular zone $(w \geq \alpha$ where $\alpha>0)$ and the regular one $(w \simeq 0)$. In [MZ99], we specify this transition by proving the existence of a profile in the variable $z=\frac{y}{\sqrt{s}}$.

THEOREM 4. Existence of a blow-up profile for equation (0.1). Assume (0.2) holds. There exists $l \in\{0,1, \ldots, N\}$ and $a N \times N$ orthogonal matrix $Q$ such that $w(Q(z) \sqrt{s}, s) \rightarrow f_{l}(z)$ uniformly on compact sets $|z| \leq C$, where $f_{l}(z)=(p-1+$ $\left.\frac{(p-1)^{2}}{4 p} \sum_{i=1}^{l}\left|z_{i}\right|^{2}\right)^{-\frac{1}{p-1}}$ if $l \geq 1$ and $f_{0}(z)=\kappa=(p-1)^{-\frac{1}{p-1}}$.

This result has been proved by Velázquez in [Vel92]. However, the convergence speed depends on the considered blow-up point in [Vel92], whereas it is uniform in [MZ99]. This uniformity allows us to derive the stability of the profile $f_{N}$ in [FKMZ].

Using renormalization theory, Bricmont and Kupiainen prove in [BK94] the existence of a solution of $(0.5)$ such that

$$
\forall s \geq s_{0}, \forall y \in \mathbb{R}^{N},\left|w(y, s)-f_{N}\left(\frac{y}{\sqrt{s}}\right)\right| \leq \frac{C}{\sqrt{s}} .
$$

Merle and Zaag prove the same result in [MZ97], thanks to a technique of finitedimension reduction. They also prove the stability of such a behavior with respect to initial data, in a neighborhood of the constructed solution. 
In [Zaa98] and [Vel92], it is proved that in this case, $u(x, t) \rightarrow u^{*}(x)$ as $t \rightarrow T$ uniformly on $\mathbb{R}^{N} \backslash\{0\}$ and that $u^{*}(x) \sim\left[\frac{8 p|\log | x \mid}{(p-1)^{2}|x|^{2}}\right]^{\frac{1}{p-1}}$ as $x \rightarrow 0$.

One interesting problem is to relate all known blow-up profiles' notions : profiles for $|y|$ bounded, $\frac{|y|}{\sqrt{s}}$ bounded or $x \simeq 0$. We prove in the following that all these descriptions are equivalent, in the case of single point blow-up with a non degenerate profile (generic case). This answers many questions which arose in former works.

THEOREM 5. Equivalence of blow-up behaviors at a blow-up point. Assume (0.2) and consider $a$ an isolated blow-up point of $u(t)$ solution of (0.1). The following behaviors of $u(t)$ and $w_{a, T}(s)$ (defined in (0.4)) are equivalent:

i) $\forall R>0, \sup _{|y| \leq R}\left|w(y, s)-\left[\kappa+\frac{\kappa}{2 p s}\left(N-\frac{1}{2}|y|^{2}\right)\right]\right|=o\left(\frac{1}{s}\right)$ as $s \rightarrow+\infty$,

ii) $\forall R>0, \sup _{|z| \leq R}\left|w(z \sqrt{s}, s)-f_{0}(z)\right| \rightarrow 0$ as $s \rightarrow+\infty$ with $f_{0}(z)=(p-1+$ $\left.\frac{(p-1)^{2}}{4 p}|z|^{2}\right)^{-\frac{1}{p-1}}$

iii) $\exists \epsilon_{0}>0$ such that for all $|x-a| \leq \epsilon_{0}, u(x, t) \rightarrow u^{*}(x)$ as $t \rightarrow T$ and $u^{*}(x) \sim\left[\frac{8 p|\log | x-a \mid}{(p-1)^{2}|x-a|^{2}}\right]^{\frac{1}{p-1}}$ as $x \rightarrow a$.

A further application of the Liouville Theorem is the stability of the behavior described in Theorem 5, with respect to perturbations in initial data. Using a dynamical system approach, we prove in [FKMZ], with Fermanian the following :

TheOREM 6. Stability of the blow-up profile. Assume (1.1) and consider $\tilde{u}(t)$ a blow-up solution of (0.1) with initial data $\tilde{u}_{0}$ which blows-up at $t=\tilde{T}$ at only one point $\tilde{a}=0$ and satisfies (0.6). Assume that

$$
\text { for all }|x| \geq R \text { and } t \in[0, \tilde{T}), \quad|\tilde{u}(x, t)| \leq M \text {. }
$$

and that the function $\tilde{w}_{0, \tilde{T}}(y, s)$ defined in (0.4) satisfies uniformly on compact sets of $\mathbb{R}^{N}$

$$
\tilde{w}_{0, \tilde{T}}(y, s)-\kappa \underset{s \rightarrow+\infty}{\sim} \frac{\kappa}{2 p s}\left(N-\frac{|y|^{2}}{2}\right) .
$$

Then, there is a neighborhood $\mathcal{V}$ in $L^{\infty}$ of $\tilde{u}_{0}$ such that for all $u_{0} \in \mathcal{V}$ the solution of (0.1) with initial data $u_{0}$ blows-up at time $T=T\left(u_{0}\right)$ at a unique point $a=a\left(u_{0}\right)$ and the function $w_{a, T}(y, s)$ defined in (0.4) satisfies uniformly on compact sets of $\mathbb{R}^{N}$

$$
w_{a, T}(y, s)-\kappa \underset{s \rightarrow+\infty}{\sim} \frac{\kappa}{2 p s}\left(N-\frac{|y|^{2}}{2}\right) .
$$

Moreover, $\left(a\left(u_{0}\right), T\left(u_{0}\right)\right)$ goes to $(0, \tilde{T})$ as $u_{0}$ goes to $\tilde{u}_{0}$.

REMARK. This results generalizes the stability result of [MZ97]. Note that unlike most applications of the Liouville Theorem, this result is valid for all subcritical $p$. In [FKZ], the same result is proved (only under the condition (0.2)), by a completely different approach based on the Liouville Theorem and on [MZ97].

REMARK. In [FKMZ], we prove the stability with respect to initial data of the blow-up behavior with the minimal speed

$$
\|u(t)\|_{L^{\infty}} \leq C(T-t)^{-\frac{1}{p-1}}
$$


for all subcritical $p$ (that is under the condition (1.1)). Note that this result is obvious under the weaker assumption (0.2), for Giga and Kohn proved in [GK87] that all blowup solutions satisfy (4.2). No blow-up rate estimate is known if

$$
u_{0} \text { has no sign and }(3 N-4) p \geq 3 N-8 .
$$

Therefore, our result is meaningful in this last case.

\section{REFERENCES}

[Bal77] J. M. BALL, Remarks on blow-up and nonexistence theorems for nonlinear evolution equations, Quart. J. Math. Oxford Ser. (2), 28 (112)(1977), pp. 473-486.

[BK94] J. BRICMONT AND A. KUPIAINEN, Universality in blow-up for nonlinear heat equations, Nonlinearity, 7(2)(1994), pp. 539-575.

[BKL94] J. BRICMONT, A. KUPIAINEN, AND G. LIN, Renormalization group and asymptotics of solutions of nonlinear parabolic equations, Comm. Pure Appl. Math., 47(6)(1994), pp. 893-922.

[CM89] X. Y. ChEN AND H. MATANo, Convergence, asymptotic periodicity, and finite-point blow-up in one-dimensional semilinear heat equations, J. Differential Equations, 78(1)(1989), pp. 160-190.

[FKMZ] C. Fermanian Kammerer, F. Merle, and H. ZAAG, Stability of the blow-up profile of non-linear heat equations from the dynamical system point of view, Math. Ann., 317 (2000), No. 2, pp. 347-387.

[FKZ] C. FERMANIAN KAMMERER AND H. ZAAG, Boundedness till blow-up of the difference between two solutions to the semilinear heat equation, Nonlinearity, 13 (2000), No. 4, pp. 11891216.

[Fri65] A. FrIEdman, Remarks on nonlinear parabolic equations, In Proc. Sympos. Appl. Math., Vol. XVII, pp. 3-23, Amer. Math. Soc., Providence, R.I., 1965.

[Fuj66] H. FujitA, On the blowing up of solutions of the Cauchy problem for $u_{t}=\Delta u+u^{1+\alpha}$, J. Fac. Sci. Univ. Tokyo Sect. I, 13 (1996), pp. 109-124.

[GK85] Y. GIGA AND R. V. KOHN, Asymptotically self-similar blow-up of semilinear heat equations, Comm. Pure Appl. Math., 38(3)(1985), pp. 297-319.

[GK87] Y. GIGA AND R. V. KoHN, Characterizing blowup using similarity variables, Indiana Univ. Math. J., 36(1)(1987), pp. 1-40.

[GK89] Y. Giga AND R. V. KoHN, Nondegeneracy of blowup for semilinear heat equations, Comm. Pure Appl. Math., 42(6)(1989), pp. 845-884.

[GV95] V. A. GalakTionov and J. L. VÁzQUez, Geometrical properties of the solutions of onedimensional nonlinear parabolic equations, Math. Ann., 303 (1995), pp. 741-769.

[HV92] M. A. Herrero AND J. J. L. VelÁzQUEZ, Flat blow-up in one-dimensional semilinear heat equations, Differential Integral Equations, 5(5)(1992), pp. 973-997.

[HV93] M. A. Herrero AND J. J. L. Velázquez, Blow-up behaviour of one-dimensional semilinear parabolic equations, Ann. Inst. H. Poincaré Anal. Non Linéaire, 10(2)(1993), pp. 131189.

[MZ97] F. MERLE AND H. ZAAG, Stability of the blow-up profile for equations of the type $u_{t}=$ $\Delta u+|u|^{p-1} u$, Duke Math. J., 86(1)(1997), pp. 143-195.

[MZ98] F. MERLE AND H. ZAAG, Optimal estimates for blowup rate and behavior for nonlinear heat equations, Comm. Pure Appl. Math., 51(2)(1998), pp. 139-196.

[MZ99] F. MERLE AND H. ZAAG, A Liouville theorem for vector-valued nonlinear heat equations and applications, Math. Ann., 316 (2000), No. 1, pp. 103-137.

[Vel92] J. J. L. VELÁZQUEZ, Higher-dimensional blow up for semilinear parabolic equations, Comm. Partial Differential Equations, 17(9-10)(1992), pp. 1567-1596.

[Zaa] H. ZAAG, A liouville theorem and blow-up behavior for a vector-valued nonlinear heat equation with no gradient structure, Comm. Pure Appl. Math., 54 (2001), No. 1, pp. 107-133.

[Zaa98] H. ZAAG, Blow-up results for vector-valued nonlinear heat equations with no gradient structure, Ann. Inst. H. Poincaré Anal. Non Linéaire, 15(5)(1998), pp. 581-622. 